\title{
ENHANCEMENT OF MAGNETIC FIELDS IN HIGH-GRAVITY STARS DUE TO AN INTRINSIC DYNAMO EFFECT
}

\author{
Dieter ENGELHARDT and Irmela BUES \\ Remeis Sternwarte, D-8600 Bamberg, Federal Republic of Germany
}

\begin{abstract}
A model of a local magnetic field within a white dwarf is constructed by the use of quantum statistics. The polarized Fermi, Bose and Maxwell statistics is calculated within a Heisenberg magnetism model by the use of Jones calculus. With a mesoscopic description the quantum mechanical dynamo can be discussed. The result is an efficient excitation mechanism, based on Lorentz time transformations and magnetic interactions. An Ap star as progenitor of a polarized white dwarf may loose its field and can generate a stronger one due to an intrinsic dynamo on a time scale of $10^{7}$ years.
\end{abstract}

\section{Introduction}

The presence of magnetic fields in white dwarfs is commonly believed to originate from their formation. Due to momentum or magnetic flux conservation the magnetic energy is concentrated within a smaller volume of the white dwarf (Chanmugam 1992). Time dependent electric fields, which may occur within the plasma, destroy the momentum conservation microscopically. Thus a large magnetic field of a white dwarf may be generated afterwards by a dynamo mechanism or by crystallization of the electrons. First we consider electrons and photons within a microscopic picture. Afterwards a mesoscopic theory is constructed, which accounts for the quantum statistics of the electrons and the partial degeneration of the plasma. The enhancement of a magnetic field within a non-degenerate plasma is discussed with possible consequences for the long-term evolution.

\section{Quantum Statistics}

The microscopic picture of the interaction of two electron states and one photon is discussed. The electron interacting with a magnetic field may be described by a Dirac spinor of $j=1 / 2$ within Euler coordinates, e.g.:

$$
\Psi_{e}=\left(\begin{array}{c}
\cos (\theta / 2) \exp (-i \Phi / 2) \\
\sin (\theta / 2) \exp (+i \Phi / 2)
\end{array}\right)
$$

where the magnetic field vector points into $\theta=0$ direction. Two electron states combine to one photon state, e.g. $\Psi_{\gamma}=\Psi_{e} * \Psi_{e}$. The quadratic expectation value 
of the momentum may be written in terms of density matrices, e.g.:

$$
\varrho_{e}=\sum_{i} \Psi_{e} * \Psi_{e}^{+} \quad, \quad \varrho_{\gamma}=\sum_{i} \Psi_{\gamma} * \Psi_{\gamma}^{+}
$$

where the matrices are averaged in terms of degenerate states $i$. The + symbol denotes Hermite conjugation of the Dirac spinor. This procedure ensures a complete description of the discrete quantum mechanical momentum of electron and photon.

The thermal Boltzmann distribution may be defined within the transfer matrix (T) formalism, e.g.: $T=\exp (-\beta H)$ where $\beta$ denotes the inverse temperature and $H$ the Heisenberg operator, e.g.(Grosse 1988):

$$
H_{i j}=J_{i j} \Psi_{i} \Psi_{j}^{+} \hbar \omega=\varrho_{i j} \hbar \omega .
$$

$J_{i j}$ denotes an effective weight of the spinor components $\Psi_{i} \Psi_{j}^{+}$and $\hbar \omega$ is the energy of the particle. The transfer matrix is used for the calculation of the expectation value of the energy with respect to the various statistics,

$$
<H>=\left(\frac{\varrho}{\exp \left(\beta \hbar \omega \varrho+\mu^{\prime}\right) \pm 1_{\text {diag }}}\right) \hbar \omega
$$

where the plus sign denotes the Fermi Dirac distribution and the minus sign the Bose statistics of the photons. $\mu^{\prime}$ is a matrix interaction term of the electrons, the chemical potential of the photons is equal to zero. For simplification we assume $\mu^{\prime} \propto \mu \varrho$, where $\mu$ is scalar.

The matrix functions are calculated within Jones calculus (1941), which was introduced for the description of photons within a crystal. The method takes advantage of the fact, that the function of a matrix must be similar to the matrix itself. It may be verified, that the function $f$ of

$$
\varrho=\left(\begin{array}{cc}
I+Q & U \\
U & I-Q
\end{array}\right)
$$

where I,Q,U are Stoke's components, may be written as

$$
f(\varrho)=1 / 2 *\left(\begin{array}{cc}
\lambda_{1}^{\prime}+\lambda_{2}^{\prime}+\left(\lambda_{1}^{\prime}-\lambda_{2}^{\prime}\right) Q / P & \left(\lambda_{1}^{\prime}-\lambda_{2}^{\prime}\right) U / P \\
\left(\lambda_{1}^{\prime}-\lambda_{2}^{\prime}\right) U / P & \lambda_{1}^{\prime}+\lambda_{2}^{\prime}-\left(\lambda_{1}^{\prime}-\lambda_{2}^{\prime}\right) Q / P
\end{array}\right)
$$

where $\lambda_{i}^{\prime}=f\left(\lambda_{i}\right)$ (scalar), $\lambda_{i}$ are two eigenvalues $\lambda_{i}=I \pm P$, and $P=\sqrt{Q^{2}+U^{2}}$ the total polarization. The complete description avoids interference effects which occur for usual solid body expansions of $\exp (\varrho)$.

We discuss now the Fermi - Dirac distribution in terms of the temperature. At low temperatures the electrons crystallize similar to the photons $\beta \rightarrow \infty:\langle H\rangle=$ $\varrho \exp (-\beta \hbar \omega \varrho)$. For $\beta \rightarrow 0$ the Maxwell limit is approached $\langle H\rangle=\varrho \hbar \omega$, e.g. the energy in terms of the Maxwell distribution is not dependent on temperature. In Fig. 1 the ferromagnetic phase transition, occuring for $\mu<0$, is visualized by a plot of the magnetization $Q$ versus temperature in terms of different $\mu$. This proves the validity of Maxwells equations. In contradiction to the boson distribution, the antisymmetric character of the wave function survives the classical limit. 


\section{Quantum Dynamics}

A complete set of mesoscopic equations is necessary for the quantum mechanical description. Within the usual MHD formalism a set of equations for the mass, the first velocity momentum, the electric and the magnetic field, the electrical current and the pressure is used. Within the quantum mechanical theory the description of the electromagnetic field $(\underline{E}, \underline{B}, \underline{j})$ is unified by the coupling of the four dimensional vector potential $A$ to the momentum of the electron. The total variation of a mesoscopic quantity may be written in a quasi classical formulation of quantum dynamics, e.g.:

$$
d \varrho / d t=i / \hbar[H, \varrho]+\partial \varrho / \partial t
$$

$\varrho$ is an arbitrary quantum mechanical operator, for example a quadratic form of wave functions. The sense of $(7)$ is justified by the validity of the temperature independent Maxwell energy.

The formalism may be used to calculate the magnetic pressure. The Hamilton operator of one Dirac electron is given by

$$
H=c \underline{\alpha} \underline{\Pi}+e A_{0}+\gamma_{4} m c^{2},
$$

where $\underline{\Pi}$ is the total momentum $\underline{\Pi}=p-e / c \underline{A}$ and $\underline{\alpha}=\gamma \gamma_{5}$ corresponds to the velocity within the classical theory written in terms of a complete set of $\gamma$ matrices. The commutator gives:

$$
i / \hbar[H, \underline{\Pi}]=e(\underline{E}+\underline{\alpha} \times \underline{B}),
$$

where the electric field is defined by the gradient of $-A_{0}$ and the magnetic field $\underline{B}=\underline{\nabla} \times \underline{A}$. The Lorentz force is to be compared to the gravitational pressure. This results in a depolarization of the momentum.

Next we consider the derivative of a magnetic field,e.g.

$$
d \underline{B} / d t=i / \hbar[H, \underline{B}]+\partial \underline{B} / \partial t
$$

$d \underline{B} / d t=0$ corresponds to van Alfvén's theorem, e.g.

$$
i / \hbar[H, \underline{B}]=-\underline{\nabla} \times(\underline{\alpha} \times \underline{B})
$$

If we consider an explicit time dependence of $\underline{B}$ as $\underline{B}(t)=\underline{B}\left(t_{1}\right) * \exp \left(i H\left(t-t_{1}\right) / \hbar\right)$ then a diffusion term $i H \underline{B}\left(t_{1}\right) / \hbar$ is found which corresponds to the Maxwell picture, since $\underline{\alpha} \underline{I}$ represents a quadratic form of the momentum operator. Therefore magnetic diffusion may occur if there are perturbations in phase.

The solution of the commutator equation without an explicit time dependence of $\underline{B}$ is well known within quantum theory, e.g.

$$
\underline{B}(t)=\exp (i H t / \hbar) \underline{B}(t=0) \exp (-i H t / \hbar) \text {. }
$$

For discussion of $\underline{B}(t)$ we add, that $H$ and $\underline{B}$ are vector spherical harmonics of rank one or may be written in terms of $\varrho_{e}$. If the matrices $H$ and $B$ are identical then the functions of $B$ and $H$ interchange and $B(t)=$ constant. If $H$ and $B$ are not 
identical, and not dependent on $\Phi$, no change in $B$ occurs either. In this case the matrices are diagonal and the matrix functions interchange. Only if one operator depends on $\Phi$, e.g. a second magnetic field interacts with $B$, the magnetic field changes. This effect is due to interferences between $Q$ and $U$, the Stoke parameter $I$ remains constant. This behaviour is similar to the $\alpha$ effect (Steenbeck et al.,1969). The expression (12) may be regarded as Lorentz transformation. The enhancement of the field is proportional to $\cosh (i H t / \hbar)^{2}$. Since this term causes an enhancement of the magnetic field if phase perturbations occur, the field may decay only due to an explicit time dependence.

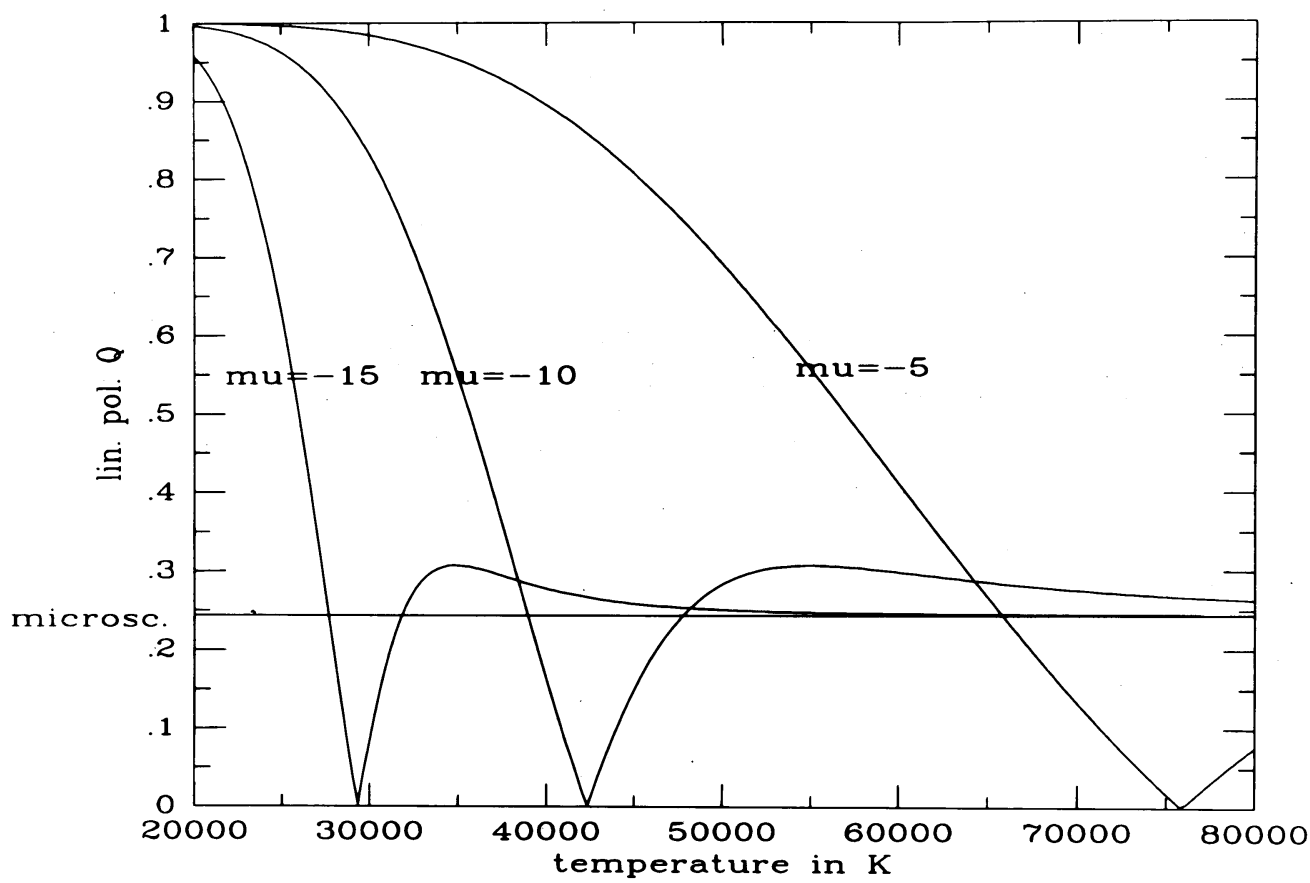

Figure 1: The magnetization $Q$ normalized to one versus temperature in terms of $\mu$. The microscopic $Q$ equals $0.245, U$ equals zero. The electrons $(20 \mathrm{eV})$ are crystallized below a critical temperature, whereas $Q$ approaches the microscopic limit very fast at higher temperatures.

\section{Discussion}

A formalism has been discussed, which describes the secular evolution of a magnetic field under the conditions of non-relativistic temperatures $T<2 * 10^{5} \mathrm{Kelvin}$ and non-crystallized electrons of positive kinetic energy. A dynamo excitation has been found, which needs a second magnetic interactor, e.g. a second dynamo for the conservation of quantum mechanical momentum.

A possible scenario of the magnetic field of a star in its Ap state and the white dwarf state may be the following: An initial magnetic field may exist in an Ap star due to a dynamo within a deeper convection zone. Mass loss may carry magnetic energy away. Shell burning may mix different volume elements and therefore a 
macroscopic magnetic field may be destroyed, since momentum is not conserved due to electric fields and the explicit time dependence of the plasma. The magnetic fields, remaining on a smaller scale, may interact with each other. By the quantum mechanical time evolution of the momentum a local twin dynamo may be excited, which leads to a strong enhancement of small scale magnetic fields and a global dynamo may be excited.

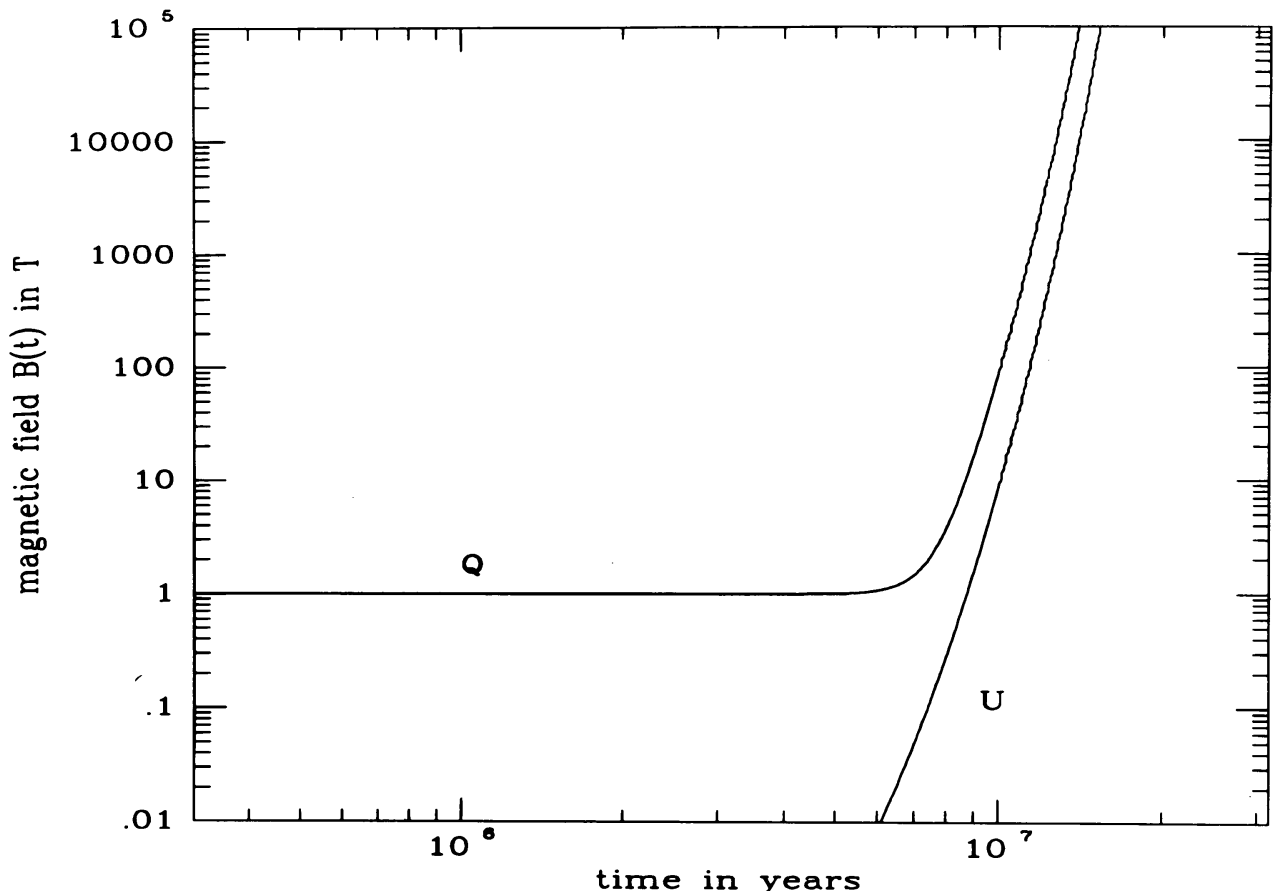

Figure 2: $|B| * Q$ and $|B| * U$ versus time. At $t=0 \quad U=0$ and $Q=1.0$, $|B(t=0)| * Q=1$ Tesla. Then the matrix $i H$ is disturbed by $\delta U=10^{-5}$ and $\delta Q=10^{-5}$. The energy per electron is $4 \mathrm{eV}$, the temperature is $20 \mathrm{kK}$ and the volume consists of $10^{10}$ electrons. When the $U$ perturbation approaches $\mathrm{Q}$ after a time scale of $10^{7}$ years, then the magnetic momentum increases very rapidly.

ACKNOWLEDGEMENTS.

This work was supported by the Deutsche Forschungsgemeinschaft with grant Bu 321/5-3.

\section{REFERENCES}

Chanmugam, G.: 1992, Ann.Rev.Astron.Astrophys. 30, 143

Engelhardt, D., Bues, I.: 1991, Lecture notes in Physics 401,428

Engelhardt, D., Bues, I.: 1992, in press

Grosse,H.:1988,'Models in Statistical Physics and Quantum Field Theory', Springer,Berlin

Jones, R.C.: 1941, J.Opt.Soc.Am. 31, 488

Landau, L.D., Lifschitz E.M.: 1986, "Quantenelektrodynamik"

Steenbeck,M., Krause, F.: 1969, Astron.Nachr. 291, 49 